\title{
ON THE EXISTENCE AND UNIQUENESS OF THE REAL LOGARITHM OF A MATRIX
}

\author{
WALTER J. CULVER ${ }^{1}$
}

1. Introduction. Consider the exponential matrix equation

$$
C=e^{x},
$$

where $C$ is a given real matrix of dimension $n \times n$. What we shall examine in this paper are the conditions under which a real matrix $X$ exists to satisfy (1.1) and, obtaining existence, the conditions under which such a solution is unique.

The significance of this study can derive from a number of sources, one of which is the mathematical modeling of dynamic systems [1].

2. A sketch of the results. According to Gantmacher [2, pp. 239$241]$, the solution to (1.1) proceeds in the following way:

We reduce $C$ to its Jordan normal form $J$ via the similarity transformation

$$
S^{-1} C S=J,
$$

whereby (1.1) becomes

$$
J=S^{-1} e^{x} S=\exp \left(S^{-1} X S\right) .
$$

We then take the natural logarithm of both sides of (2.2) and invert the similarity transformation to obtain the desired solution(s) $X$.

As we will show rigorously, a real solution exists provided $C$ is nonsingular and each elementary divisor (Jordan block) of $C$ corresponding to a negative eigenvalue occurs an even number of times. This assures that the complex part of $X$ will have complex conjugate elementary divisors (Jordan blocks).

The possible nonuniqueness of the solution can arise in two ways as we will demonstrate: (1) because the matrix $C$ has complex eigenvalues and hence provides $\log J$ with at least a countable infinity of periodic values, and (2) because the similarity transformation which relates $J$ to $C$ uniquely $^{2}$ via (2.1) may not relate $\log J$ to $X$ uniquely via (2.2), in which case an uncountable infinity of solutions results.

Received by the editors August 28, 1964 and, in revised form, March 31, 1966.

1 Surface Division, Westinghouse Defense and Space Center, P.O. Box 1897, Baltimore 3, Maryland.

2 A Jordan form $J$ is unique to within an ordering of diagonal blocks. 
Case (2) corresponds to the situation where $\log J$ cannot be expressed as a power series in $J$.

3. The mathematical preliminaries. It is well known that the matrix $S$ in (2.1) is not unique, although $J$ is uniquely related to $C$. In this regard, the following lemma is of interest.

LEMмA 1. Every matrix $S$ which takes a given matrix $C$ into its Jordan form $J$ via the relation

$$
C=S J S^{-1},
$$

differs from any other matrix $\widetilde{S}$ which does the same thing, i.e.,

$$
C=\tilde{S} J \widetilde{S}^{-1}
$$

only by a multiplicative nonsingular matrix factor $K$ which is one of a continuum of such matrices that commute with $J$ and provide the identity

$$
\tilde{S}=S K \text {. }
$$

Proof. Equate (3.1) to (3.2) and rearrange terms to obtain

$$
\left(S^{-1} \tilde{S}\right) J=J\left(S^{-1} \tilde{S}\right)
$$

From this it is obvious that $S^{-1} \tilde{S}$ must be a matrix, say $K$, which commutes with $J$, and is nonsingular, wherefrom (3.3) follows directly to complete the proof of the lemma.

Clearly, now, if $S$ is replaced by the more general transformation $\tilde{S}=S K$, equations (2.1) and (2.2) remain exactly the same, since every $K$ commutes with $J$. However, after the logarithm of $J$ is taken, $K$ may not commute with $\log J$, so that for complete generality we must write

$$
X=S K(\log J) K^{-1} S^{-1} .
$$

The logarithm of $J$ is well defined [2, p. 100] in terms of its real Jordan blocks $J_{1}, \cdots, J_{m}, m \leqq n$ :

$$
\log J=\operatorname{diag}\left\{\log J_{1}, \cdots, \log J_{m}\right\} .
$$

Typically, if the $k$ th block is of dimension $\left(\alpha_{k}+1\right) \times\left(\alpha_{k}+1\right)$ and corresponds to the real elementary divisor

$$
\left(\lambda-\lambda_{k}\right)^{\alpha+1},
$$

where $\lambda_{k}$ is a real eigenvalue of $C$ not necessarily different from $\lambda_{h}(h \neq k)$, then 


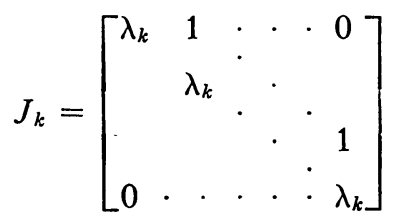

and

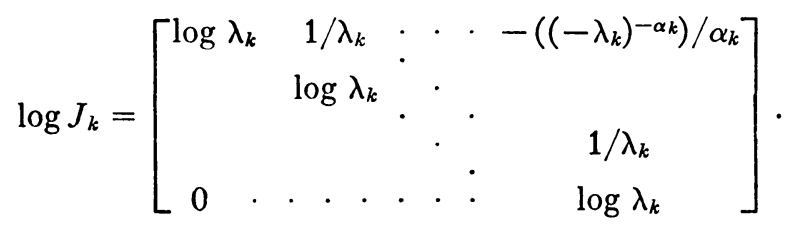

If, on the other hand, the $k$ th block corresponds to the complex conjugate elementary divisors

$$
\left(\lambda-\lambda_{k}\right)^{\beta_{k}+1} \text { and }\left(\lambda-\lambda_{k}^{*}\right)^{\beta_{k}+1},
$$

where $\lambda_{k}=u_{k}+i v_{k}$ is a complex eigenvalue of $C$ and $\lambda_{k}^{*}$ is its complex conjugate, then the block dimensions are $2\left(\beta_{k}+1\right) \times 2\left(\beta_{k}+1\right)$ and

$$
J_{k}=\left[\begin{array}{cccccc}
L_{k} & I & \cdot & \cdot & \cdot & 0 \\
& L_{k} & \cdot & & \\
& & \cdot & \cdot & \\
& & & & \cdot & I \\
0 & & & & & L_{k}
\end{array}\right],
$$

where

$$
L_{k}=\left[\begin{array}{rr}
u_{k} & -v_{k} \\
v_{k} & u_{k}
\end{array}\right]
$$

For this complex case,

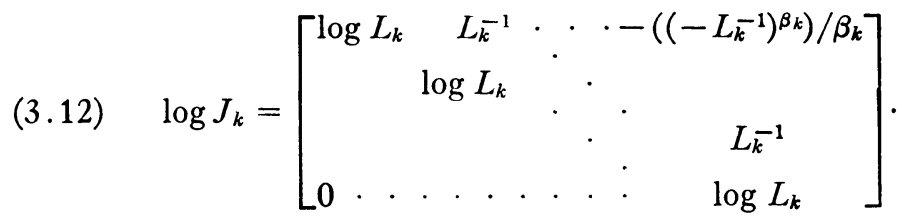

Since all matrix logarithms are defined ultimately by the matrix exponential, e.g.,

$$
J_{k}=\exp \left(\log J_{k}\right),
$$

it follows that such logarithms are multivalued functions of the type

$$
\log J_{k}=\operatorname{LOG} J_{k}+D \text {, }
$$


where LOG is the principal value and $D$ is one of an infinity of matrices that commute with LOG $J_{k}$ and satisfy the relation $e^{D}=I$.

The nature of $D$ depends on whether the $\lambda_{k}$ belonging to $J_{k}$ is real or complex. If $\lambda_{k}$ is real, the eigenvalues of $\log J_{k}$ are its diagonal elements, and from a theorem in Gantmacher [2, p. 158], these must be equal. Thus

$$
\log J_{k}=\operatorname{LOG} J_{k}+i 2 \pi q_{k} I, \quad \lambda_{k} \text { real, }
$$

where $q_{k}=0, \pm 1, \pm 2, \cdots$.

On the other hand, if $\operatorname{Im} \lambda_{k} \neq 0$, the real and imaginary parts of the eigenvalues of $\log J_{k}$ appear respectively on the main and skew diagonals of the $2 \times 2$ diagonal blocks of $\log J_{k}$. Again Gantmacher's theorem can be used, this time to infer that the diagonal blocks of $\log J_{k}$ must be equal. Thus

$$
\log J_{k}=\operatorname{LOG} J_{k}+2 \pi\left(i q_{k} I+r_{k} E\right), \quad \operatorname{Im} \lambda_{k} \neq 0,
$$

where both $q_{k}$ and $r_{k}$ can assume the values $0, \pm 1, \pm 2, \cdots$, and where

$$
E=\operatorname{diag}\left\{\left[\begin{array}{rr}
0 & -1 \\
1 & 0
\end{array}\right], \cdots,\left[\begin{array}{rr}
0 & -1 \\
1 & 0
\end{array}\right]\right\} \text {. }
$$

4. Development of results. From expressions (3.14) and (3.15) we can see that if no constraints are put on the solution $X=$ $S K\left(\operatorname{diag}\left\{\log J_{1}, \cdots, \log J_{m}\right\}\right) K^{-1} S^{-1}$, then at least a countable infinity of $X$ 's are produced. In this paper we apply, for physical reasons [1], the constraint that $X$ be real, the immediate consequence of which is that the complex elementary divisors (Jordan blocks) of $X$ must appear in complex conjugate pairs. The question of existence under this constraint is answered by the following theorem.

Theorem 1. Let $C$ be a real square matrix. Then there exists a real solution $X$ to the equation $C=e^{X}$ if and only if $(*) C$ is nonsingular and each elementary divisor (Jordan block) of $C$ belonging to a negative eigenvalue occurs an even number of times.

Proof. $^{3}$ (i) Necessity. Let $X$ be real such that $C=e^{x}$. If any complex eigenvalues of $X$ exist, they must correspond to complex conjugate elementary divisors. Hence, we may suppose that the elementary divisors of $X$ are

$$
\begin{aligned}
& \left(\lambda-z_{k}\right)^{a_{k}}, z_{k} \text { real, } \\
& \left(\lambda-z_{k}\right)^{b_{k}} \text { and }\left(\lambda-z_{k}^{*}\right)^{b_{k}}, \quad \operatorname{Im} z_{k} \neq 0 .
\end{aligned}
$$

8 The proof in this form is due essentially to the reviewer of the paper. 
Since $d e^{\lambda} / d \lambda \neq 0$ for all finite $\lambda$, it follows from a theorem in Gantmacher $\left[2\right.$, p. 158] that the elementary divisors of $C=e^{\boldsymbol{X}}$ are

$$
\begin{aligned}
& \left(\lambda-e^{z k}\right)^{a_{k}}, \quad z_{k} \text { real } \\
& \left(\lambda-e^{z_{k}}\right)^{b_{k}} \text { and }\left(\lambda-e^{z_{k}^{*}}\right)^{b_{k}}, \quad \operatorname{Im} z_{k} \neq 0 \text {. }
\end{aligned}
$$

In no event is $e^{z_{k}}=0$. Moreover, $e^{z_{k}}<0$ only if $\operatorname{Im} z_{k} \neq 0$, in which case $e^{z k}=e^{z k^{*}}$. Thus the negative eigenvalues of $C$ must associate with elementary divisors which occur in pairs. Hence $C$ must satisfy (*).

(ii) Sufficiency. Conversely, let $C$ satisfy $(*)$. Its eigenvalues $\lambda_{k}$ are as specified by (3.6) or (3.9). For those $\lambda_{k}$ that are real and negative we can write $\lambda_{k}=e^{z k}=e^{z k^{*}}$, where $z_{k}=\mathrm{LOG}\left|\lambda_{k}\right|+i \pi$. Moreover, by the last part of $(*)$, the corresponding elementary divisors are $\left(\lambda-e^{z k}\right)^{\alpha_{k}+1}$ and $\left(\lambda-e^{z k^{*}}\right)^{\alpha_{k}+1}$. Since, also, $C$ is real, we may suppose that all the elementary divisors of $C$ are given by (4.2). Consider, now, the class of matrices with elementary divisors (4.1). Clearly there exists some real matrix $Y$ in this class. By the theorem quoted from Gantmacher, the function $e^{Y}$ must be similar to $C$, so that a real matrix $T$ can be found such that

$$
C=T^{-1} e^{Y} T=\exp \left(T^{-1} Y T\right) .
$$

Identify $X$ with $T^{-1} Y T$ to confirm the sufficiency of $(*)$.

THEOREM 2. Let $C$ be a real square matrix. Then the equation $C=e^{X}$ has a unique real solution $X$ if and only if (**) all the eigenvalues of $C$ are positive real and no elementary divisor (Jordan block) of $C$ belonging to any eigenvalue appears more than once.

Proof. (i) Sufficiency. All the solutions to $C=e^{x}$ are given by (3.4), (3.5):

$$
X=S K\left(\operatorname{diag}\left\{\log J_{1}, \cdots, \log J_{m}\right\}\right) K^{-1} S^{-1},
$$

where $\log J_{k}$ is given by (3.14) or (3.15). Clearly, if $(* *)$ holds, LOG $J_{k}$ is real, whereas $\log J_{k}=$ LOG $J_{k}+i 2 \pi q_{k} I$ is complex and has no complex conjugate in the set $\log J_{h}=\mathrm{LOG} J_{h}+i 2 \pi q_{h} I, h \neq k$. Hence, for every $k$ the parameter $q_{k}$ must be zero, and for every set of blocks (say $J_{k}, J_{k+1}, \cdots, J_{k+\gamma_{k}}$ ) which belongs to the eigenvalue $\lambda_{k}$ there exists the unique set LOG $J_{k}$, LOG $J_{k+1}, \cdots$, LOG $J_{k+\gamma_{k}}$ which belongs to the eigenvalue LOG $\lambda_{k}$. Hence [2, p. 220] every $K$ that commutes with $J$ must also commute with $\log J$ in (3.4), and $(* *)$ is sufficient for $X$ to be real and unique.

(ii) Necessity. Take the contradictions to $(* *)$ which satisfy condition (*) of Theorem 1. For example, assume $C$ to have positive real 
eigenvalues which belong to Jordan blocks that appear more than once, or assume $C$ to have negative real eigenvalues (whose blocks must occur in pairs), or assume $C$ to have complex conjugate eigenvalues.

Suppose, first, that $\lambda_{k}$ is real and corresponds to the identical blocks $J_{k}, J_{k+1}$. If in (3.14) we choose $q_{k}=-q_{k+1}$ for $\lambda_{k}$ positive real and $q_{k}=-\left(1+q_{k+1}\right)$ for $\lambda_{k}$ negative real, we obtain the complex conjugate blocks $\log J_{k}, \log J_{k+1}$. Hence a continuum set of $K$ matrices that commuted with $J$ will not commute with $\log J$, and a continuum of real $X$ 's will arise from (3.4).

Suppose now, that some pair of eigenvalues of $C$ are complex conjugate. If they correspond to Jordan blocks that appear more than once (say $J_{k}, J_{k+1}$ ), then by taking $q_{k}=-q_{k+1}$ in (3.15) we obtain a continuum set of $X$ 's from (3.4). If the blocks appear only once, $q_{k}$ must be zero for all $k$ or else $\log J_{k}$ will be a complex block without a conjugate. However, $r_{k}$ in (3.15) can be any integer. If any two blocks (say $J_{k}, J_{k+1}$ ) are not identical but belong to the same eigenvalue $\lambda_{k}$, the fact that $r_{k}$ need not equal $r_{k+1}$ makes it possible for $\log J_{k}$ and $\log J_{k+1}$ to belong to different eigenvalues. Hence not every $K$ will commute with $\log J$ and again a continuum of $X$ 's result. Finally, if no two blocks of $J$ belong to the same complex eigenvalue, every $K$ that commutes with $J$ will also commute with $\log J$, provided the Jordan blocks for the real eigenvalues appear only once. But $r_{k}$ can still be any integer, which leads to a countable infinity of $\log J$ 's, and hence to a countable infinity of $X^{\prime}$ 's. Thus (**) is necessary.

Corollary. Let $C$ be a real square matrix and let $C=e^{x}$ have more than one real solution $X$. Then there exists an infinity of real solutions $X$ which are

(a) Countable if all real eigenvalues of $C$ are positive such that their Jordan blocks appear only once and $C$ has complex eigenvalues none of which belongs to more than one Jordan block;

(b) Uncountable if any real eigenvalues of $C$ are negative, or if any positive real eigenvalues belong to Jordan blocks that appear more than once, or if any complex conjugate eigenvalues belong to more than one Jordan block.

\section{REFERENCES}

1. W. J. Culver, An analytic theory of modeling for a class of minimal-energy control systems (disturbance-free case), SIAM J. Control 2 (1964), 267-294.

2. F. R. Gantmacher, The theory of matrices, Vol. I, Chelsea, New York, 1959. 